\title{
The European initiative for the development of Infrastructural Tools: the INTOOL programme.
}

\section{P.COUSIN}

European Project Officer for the European Commission and EOTC; Technical Director - CETECOM France

2, rue Jacques Monod - F-91893 Orsay Cedex France

Tel:+33169351313 Fax: +33169351314; cousinph@aol.com

\begin{abstract}
This paper presents the INTOOL programme of the European Commission which gave financial support to the development of Infrastructural Tools. Its aim was to improve the quality of test system components, to speed up and to reduce the costs of global test services development process in Information Technology and Telecommunications within Europe. This initiative, of a horizontal nature, was the political and technical follow up of the multiple sectoral support given by the CTS (Conformance Testing Services) programme during 12 years and involving more than 100 Million Ecus. Details are given on the political and technical background which gave justification to the launching of the INTOOL programme in 1994. The achievements of the programme are presented.
\end{abstract}

\section{Keywords}

European Commission, generic, Intool, CATG, compiler, open architecture 


\section{INTRODUCTION}

Increasingly there is a drive to make communications protocol testing more costeffective. One facet of this is to increase the use of automated tools in an efficient manner. Early tools tended to be created as bespoke software to solve a specific problem. However, the market for specific test tools is rather limited and insufficient to justify the high cost of these early developments. Thus, there is now a strong movement in the market towards using more generic tools which can be tailored to solve a whole range of problems.

What is tending to happen at present is that the dominant tool suppliers are creating their own suites of generic tools which can be combined in many different ways but only with tools from the one supplier. Different suites of tools from different suppliers remain incompatible. Thus, test tool users are getting locked into using tools from a single supplier and this is hindering the development of a truly competitive test tool market in Europe. Users want to be able to select the best tools for the job with the confidence that different tools from different suppliers can be used in combination.

In order firstly to have a genuinely open and competitive test tools market and secondly to have maximum flexibility in using automated tools in communications testing (whether for conformance testing or interoperability testing or both), it is desirable to be able to select different tools from different suppliers and use them in combination. In order to ensure this, it is necessary to have agreed specifications for key interfaces between different tools.

To address these issues the European Commission launched the Infrastructural Tool programme (INTOOL) and have been supporting three INTOOL projects during 1995 and 1996 in order to support the development of generic tools to facilitate the use of automation in the testing infrastructure in Europe.

\section{BACKGROUND}

The achievement of a single European market will increasingly rely on the removal of technical barriers to trade.

Since 1983, considerable efforts have been made by the European Community to develop one of the key ingredients required to promote the objectives of economic integration in this field : a standardisation policy aimed at opening the markets to the free circulation of goods and the implementation of trans-European services.

Much progress has been accomplished in the area of European standardisation for several industrial sectors by the European Standardisation Organisations (ESO: CEN-CENELEC-ETSI). At the same time, it was apparent from early on 
that testing and certification had a vital role to play as a necessary complement to standardisation actions if standards were to be implemented in practice.

Testing and certification represent an essential component of Community standardisation policy. In many fields the complexity of standards is magnified to a degree where it becomes difficult, if not impossible, to implement the standards without creating technical divergence which will ultimately result in a lack of interworking or a mismatch to the initial specifications. Therefore, the need for an adequate guarantee that products conform to standards- to unique interpretation of standards- emerges as a decisive condition for building up confidence in the standardised products.

Consequently, in 1985, the Commission of European Communities launched the conformance test services (CTS) programme, covering only the IT\&T field to provide tools and facilities to meet the growing market for truly interoperable IT\&T systems.

The basic idea of the CTS programme was to establish real testing services for the market, capable of verifying the conformity of products to the reference standards, based on the principle of a standard testing methodology thus leading to comparability of results and, eventually, mutual recognition of test reports and certificates. The organisations providing the services are called testing centres or testing laboratories.

Since 1985, six calls for proposals have been launched to invite interested and qualified organisations to set up testing service at reduced risk (50\% contribution), each call resulting in a set of new projects launched for an average duration of 30 months.

The current map of the CTS programme includes 50 testing centres offering testing services across Europe for about 60 technical areas. Each service is offered by at least two centres in Europe: although only two are funded, often the number of centres offering the same service is larger. The CTS experience, however, involving, as it did, multiple contacts with relevant technical committees of the ESOs, as well as the members of the testing community, has highlighted the following problems which were not fully addressed within the CTS programme:

- INTERPRETATION AND IMPLEMENTATION OF STANDARDS: the development process of standards does not always make it possible to guarantee a rapid and effective implementation of the conformity assessment procedures against these standards under optimum economic conditions.

- COST AND TIME REQUIRED TO DEVELOP NEW TOOLS: the development cost of new tools is too high and all the more so where the product is integrated and has hidden functions. The deadlines for their development are often too long.

- NECESSARY NEW THINKING ON METHODS: the product validation methods in certain complex fields still remain confined to the field of theoretical studies.

Bearing these aspects in mind, in 1992, the EC launched a study where the objective was to analyse the different steps between the availability of standards and the availability of maintainable conformance testing services for that 
standard. The main result was the definition of a model identifying processes, key entities and areas where the application of productivity tools may contribute to improve efficiency and economy.

Following such a line, in June 1994, the EC gave support to the development and delivery of infrastructural tool(s) generally accessible within Europe with the aims of improving the quality of test system components and/or speeding up the global and reducing the cost of test services development process and/or facilitating the establishment of conformance testing services in Information Technology and Telecommunications.

The call for proposals was limited to a well defined list of domains.

\section{- CATG : Computer Aided Test Generation; \\ - GCI: Generic Compiler or Interpreter; \\ - OTE: Open Test Environment.}

Three projects involving several European companies were established. They terminated in March 1997 and the public results are now available. A free CDROM gathering all the information and giving the public domain specifications is available on demand from the author of this paper.

\section{THE CATG PROJECT}

It is apparent from many different sources that there is a great deal of work to do to produce a complete set of Abstract Test Suites from a base standard or specification notation starting point. Once these are produced, there is a considerable ongoing maintenance requirement. As in many other areas, computer technology may be applied to increase efficiency and effectiveness. Years of experience in the domain of test suite generation in TTCN(Tree and Tabular Combined Notation) and in SDL (Specification Description Language) have been concretised in the CATG project which put a commercial tool on the market.

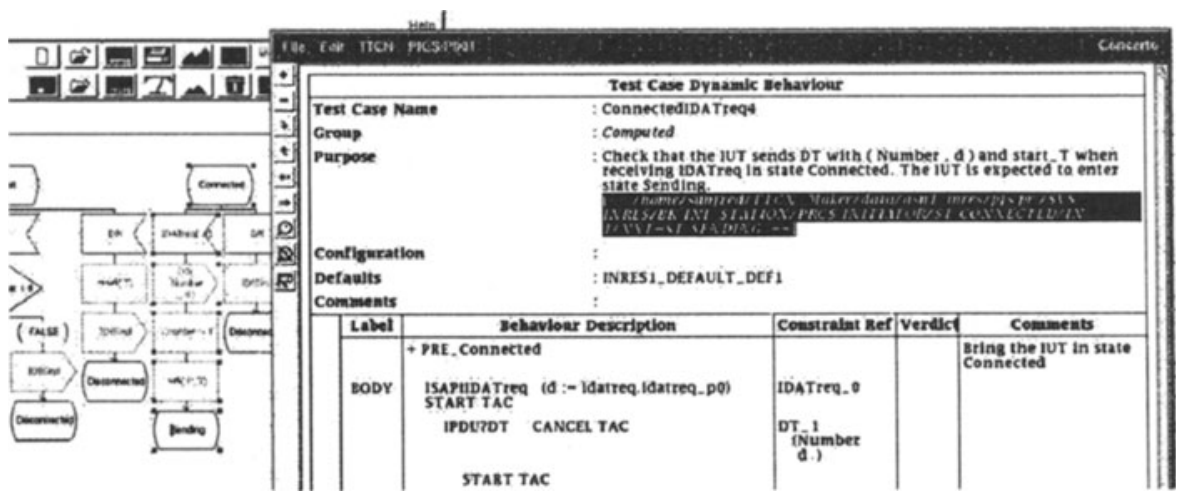


Figure 1 Exemple of TTCN Test Case generated from SDL description.

\section{THE GCI PROJECT}

This project worked on a model which would help to develop TTCN compilers, the final objective being to directly implement standards coming from standardisation bodies already drafted in TTCN into test systems.

Existing TTCN test systems can normally be thought of as containing two parts; one that handles the interpretation and execution of the TTCN, and one that adapts this TTCN execution for use with the particular system under test. The intention of the GCI interface is to allow the separation of these parts, so that the same TTCN compiler/interpreter can be reused in a number of different test systems

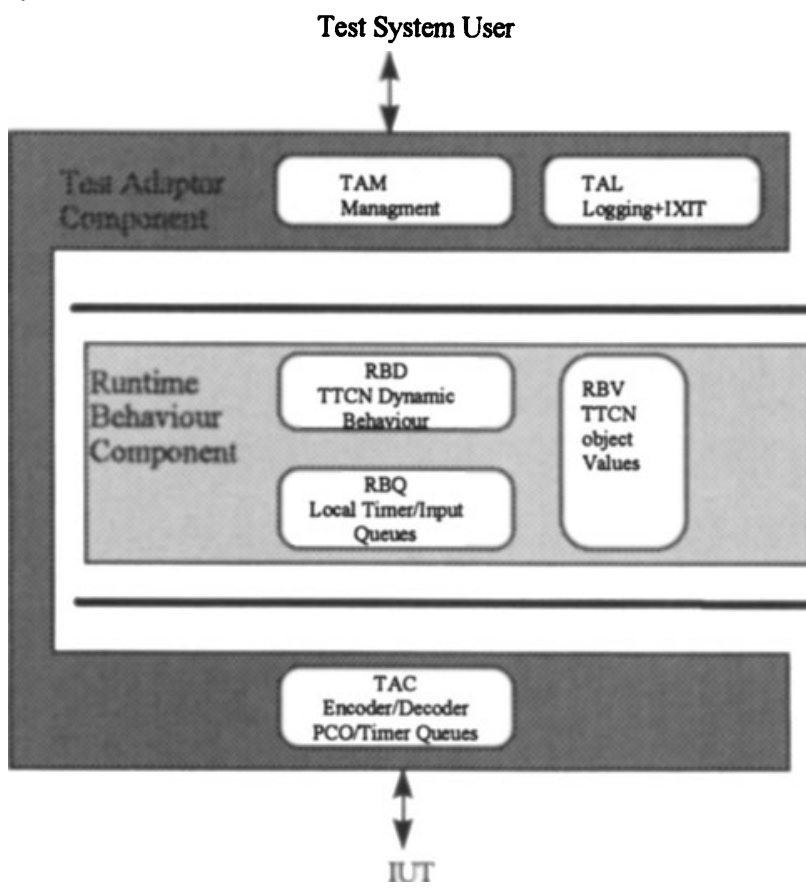

Figure 2 The GCI Interface model.

\section{THE OTE PROJECT}

The basic idea was to define an open architecture where generic reusable components and existing dedicated products can co-operate. The project has provided a set of specifications (OTE architecture, objects, communications 
protocols) as well as commercially available tools. The OTE Architecture is an open environment dedicated to the distributed test process. It includes the interface to access and manage the OTE objects and the interface for tool communication. The architecture can be implemented with several technologies (Corba, Proprietary solutions etc). Objects Specifications offer a common format for the objects involved in testing. Interchange of information between the actors of the test process is easy through OTE. OTE Piloting Protocol (PMI) is the powerful solution to control several test equipment involved simultaneously in distributed testing. PMI covers all the phases of the test process and the aspects of remote control of the test equipment.

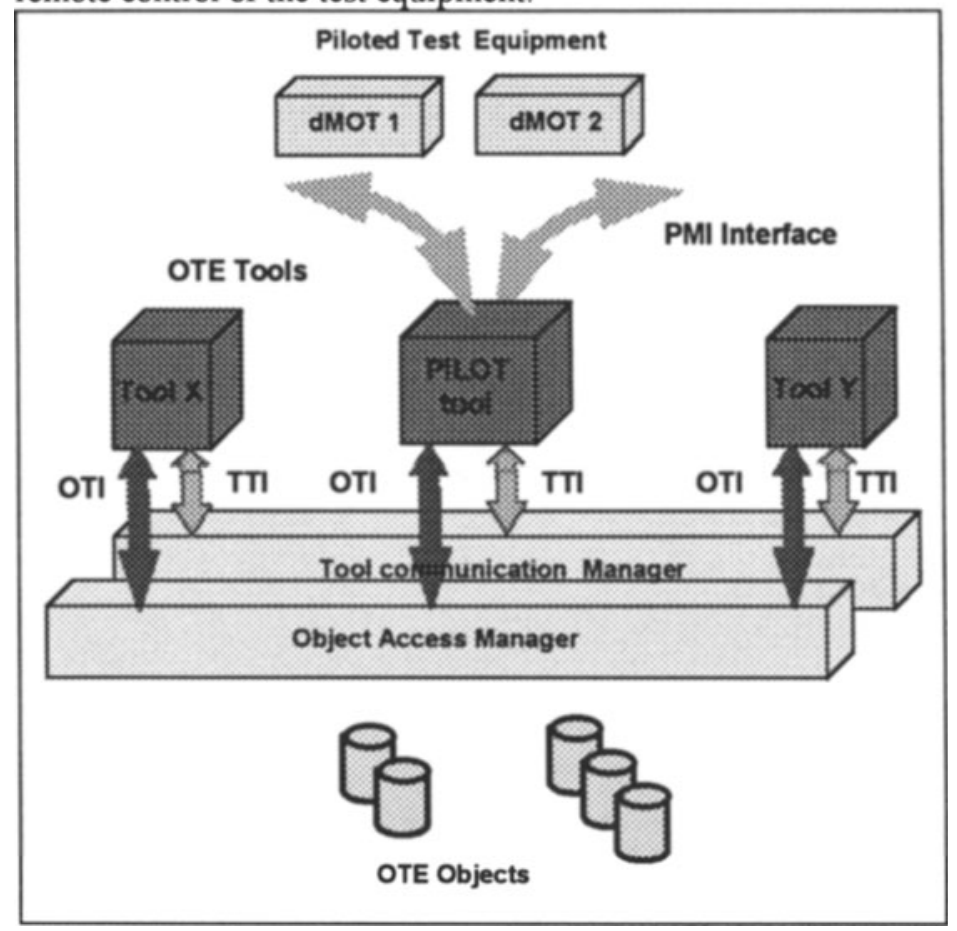

Figure 3 the OTE architecture.

\section{BIOGRAPHY}

Philippe COUSIN - Technical Director - CETECOM France

15 years experience in Telecommunications, Research and Testing including in the past 5 years a broad insight into ICT and other industrial matters at the European policy-making level at the European Commission and EOTC (European Organisation for Testing and Certification). EC and EOTC Project Officer of CTS and INTOOL projects. Several years of operational activities including within a 
testing laboratory and research activities in the development of testing facilities and methodologies (TTCN, SDL, ISO9646). Representative of France Telecom and European Commission in Standardisation bodies and pre-normative various working groups. Project Leader of Standardisation Project teams. 